\title{
BMJ Open Protocol for economic evaluation alongside the SHINE (Supporting Healthy Image, Nutrition and Exercise) cluster randomised controlled trial
}

\author{
Victoria Brown (1) , ${ }^{1}$ Joanne Williams, ${ }^{2}$ Lisa McGivern, ${ }^{2}$ Susan Sawyer, ${ }^{3}$ \\ Liliana Orellana, ${ }^{4}$ Wei Luo, ${ }^{5}$ Kylie D Hesketh, ${ }^{6}$ Denise E Wilfley, ${ }^{7}$ Marj Moodie ${ }^{1}$
}

To cite: Brown V, Williams J, McGivern L, et al. Protocol for economic evaluation alongside the SHINE (Supporting Healthy Image, Nutrition and Exercise) cluster randomised controlled trial. BMJ Open 2020;10:e038050. doi:10.1136/ bmjopen-2020-038050

- Prepublication history for this paper is available online. To view these files, please visit the journal online (http://dx.doi org/10.1136/bmjopen-2020038050).

Received 25 February 2020 Revised 10 May 2020 Accepted 29 May 2020

Check for updates

(C) Author(s) (or their employer(s)) 2020. Re-use permitted under CC BY-NC. No commercial re-use. See rights and permissions. Published by BMJ.

For numbered affiliations see end of article.

Correspondence to

Dr Victoria Brown;

victoria.brown@deakin.edu.au

\section{ABSTRACT}

Introduction Limited evidence exists on the costeffectiveness of interventions to prevent obesity and promote healthy body image in adolescents. The SHINE (Supporting Healthy Image, Nutrition and Exercise) study is a cluster randomised control trial (CRCT) aiming to deliver universal education about healthy nutrition and physical activity to adolescents, as well as targeted advice to young people with body image concerns who are at risk of developing disordered eating behaviours. This paper describes the methods for the economic evaluation of the SHINE cRCT, to determine whether the intervention is costeffective as an obesity prevention measure.

Methods and analysis A public payer perspective will be adopted, with intervention costs collected prospectively. Within-trial cost-effectiveness analysis (CEA) and costutility analysis (CUA) will quantify the incremental costs and health gains of the intervention as compared with usual practice (ie, teacher-delivered curriculum). CEA will present results as cost per body mass index unit saved. CUA will present results as cost per quality-adjusted life year gained. A modelled CUA will extend the target population, time horizon and decision context to provide valuable information to policymakers on the potential for incremental cost offsets attributable to disease prevention arising from intervention. Intervention costs and effects will be extrapolated to the population of Australian adolescents in Grade 7 of secondary school (approximate age 13 years) and modelled over the cohort's lifetime. Modelled CUA results will be presented as health-adjusted life years saved and healthcare cost-savings of diseases averted. Incremental costeffectiveness ratios will be calculated as the difference in costs between the intervention and comparator divided by the difference in benefit. Semi-structured interviews with key intervention stakeholders will explore the potential impact of scalability on cost-effectiveness. These data will be thematically analysed to inform sensitivity analysis of the base case economic evaluation, such that costeffectiveness evidence is reflective of the potential for scalability.

Ethics and dissemination Ethics approval was obtained from the Deakin University Human Research Ethics Committee (\#2017-269) and the Victorian Department of Education and Training (\#2018_003630). Study findings will be disseminated through peer-reviewed academic
Strengths and limitations of this study

- This protocol contributes to the limited body of evidence on the cost-effectiveness of interventions that prevent obesity and promote healthy body image in adolescents.

- Data collection for the economic evaluation is prospectively planned alongside the randomised controlled trial that will assess intervention effectiveness.

- The cost-effectiveness study design will generate important information for decision-makers, providing evidence of the immediate 'value-for-money' of the intervention.

- A modelled economic evaluation will estimate the long-term potential for cost-effectiveness of the intervention as an obesity prevention measure.

- Qualitative exploration of the potential impacts of the programme delivered at scale on costs, effects and cost-effectiveness will provide important information on scalability of the intervention.

papers and participating schools will receive annual reports over the 3 years of data collection.

Trial registration number ACTRN 12618000330246;

Pre-results.

\section{INTRODUCTION}

Overweight and obesity in adolescence is a serious public health issue globally, with both the mean body mass index (BMI) and the prevalence of obesity in adolescents increasing worldwide over the last four decades. $^{1}$ In Australia, approximately 30\% of boys and $24 \%$ of girls aged 15 to 17 years were classified as overweight or obese in 2017/2018. ${ }^{2}$ Diet, physical activity and sedentary behaviour are recognised as modifiable determinants of obesity at the individual level, yet evidence suggests that many adolescents do not regularly engage in healthy behaviours to reduce their risk of overweight and obesity. For instance, in 2011/2012, less 
than $10 \%$ of Australian adolescents aged 12 to 17 years met recommended daily physical activity guidelines ${ }^{3}$ and recent evidence suggests that Australian children aged 14 to 18 years consume relatively high amounts of discretionary food, including cakes, biscuits, fried potatoes and sugar-sweetened beverages. ${ }^{4}$

Overweight and obesity are associated with higher healthcare costs and greater health burden within childhood and adolescence, including negative psychosocial consequences, lower educational attainment and increased risk of sleep apnoea, hypertension, type 2 diabetes and asthma. ${ }^{15-7}$ Evidence also suggests that overweight and obesity in childhood and adolescence tracks to adulthood, ${ }^{8}$ leading to adverse health consequences throughout the life course related to chronic diseases where excess body weight is a risk factor. ${ }^{9}$ Adolescence has been identified as a period of vulnerability, when many disordered eating behaviours and attitudes that lead to weight gain emerge. ${ }^{10}$ The adolescent years introduce greater autonomy over food choice, a decrease in breakfast consumption, increased frequency of snacking and eating outside the home environment, more disposable income, stronger influence from peers and changes in physical activity behaviours. ${ }^{11}$ Adolescence may therefore present a window for intervention to reduce body weight and image concerns, and to improve nutrition and physical activity behaviours.

The evidence for the effectiveness of obesity prevention interventions in adolescents (ie, children aged 13 to 18 years) is currently limited and relatively inconclusive. ${ }^{12} 13$ An important finding from a recent Cochrane review was that interventions to prevent childhood obesity do not appear to result in adverse effects. ${ }^{13}$ Evidence suggests that internet-delivered interventions can reduce weight and shape concerns in adolescents, ${ }^{14}{ }^{15}$ however limited evidence exists on the cost-effectiveness of such interventions. There is also extremely limited evidence to date on the cost-effectiveness of interventions to reduce body weight and encourage healthy nutrition and physical activity in adolescents. ${ }^{16}$ Information on the cost-effectiveness of interventions is an important element in the decisionmaking process, allowing for more informed resource allocation decisions in environments where there are infinite wants and needs for health spending yet finite resources in terms of funding allocated to health. Cost-effectiveness evidence is often informed by data from controlled research environments such as randomised controlled trials (RCTs), however, there is also a need to better understand the potential impact of the scale-up of interventions on the costs, effects and cost-effectiveness of obesity prevention interventions when delivered in the 'real world'. 1617 Qualitative methods for economic evaluation have recently been highlighted as promising for better understanding of the contextual factors of implementation (eg, organisational, environmental, economic factors) that may be relevant to decision-makers. ${ }^{18}$ These methods have not yet been comprehensively applied to economic evaluations of interventions conducted in controlled research settings to provide valuable information on the impacts of scale-up in less controlled environments.

The SHINE (Supporting Healthy Image, Nutrition and Exercise) study is a cluster RCT (cRCT), with randomisation at the school level. SHINE aims to deliver universal education about healthy nutrition, physical activity and well-being behaviours to adolescents, as well as targeted advice to young people who may be experiencing or are at risk of developing mental health, weight or body image disorders. The intervention replaces the usual Health and Physical Education (HPE) curriculum for 8 weeks and is a self-directed, online learning programme that is modelled on cognitive behavioural therapy (CBT) to effect change. ${ }^{19}$ The intervention aims to improve body image, mental health, nutrition and physical activity and to help prevent the development or progression of overweight and obesity. The aim of this paper is to describe the protocol for the economic evaluation of the SHINE cRCT. The evaluation will address the research question of whether the SHINE intervention is cost-effective for reducing BMI and increasing physical activity, as compared with usual practice. The economic considerations of future scale-up of the intervention will also be explored using qualitative methods. Combined, this will provide important information on the cost-effectiveness of the intervention and its potential for wider implementation across Australian secondary schools.

\section{METHODS AND ANALYSIS \\ Design overview}

Within-trial cost-effectiveness analysis (CEA) and costutility analysis (CUA) will be undertaken alongside the SHINE study, quantifying the incremental costs and health gains of the intervention as compared with usual practice (ie, teacher-delivered curriculum). Decision-analytical modelling will then extend the target population, time horizon and decision context from the within-trial results, to provide valuable information to policymakers on the potential for incremental cost offsets attributable to disease prevention as a result of the intervention. CEA and CUA will be conducted at 36 months using an intention-to-treat approach. Cost-effectiveness of the SHINE intervention will be determined using the commonly accepted Australian threshold of \$A 50000 per quality-adjusted life year (QALY) gained..$^{20} \mathrm{~A}$ qualitative study will also explore the economic implications of scale-up on the costs, effects and cost-effectiveness of the SHINE intervention should it be implemented at scale. Reporting of the economic evaluation will follow the Consolidated Health Economic Evaluation Reporting Standards (CHEERS) ${ }^{21}$ and a CHEERS checklist will be completed.

\section{Study population}

Trial participants will be Grade 7 students (approximately 13 years of age) attending 30 secondary schools across metropolitan Melbourne and country Victoria, Australia. 


\begin{tabular}{|c|c|c|c|c|}
\hline & $\begin{array}{c}2019 \\
\text { (Year 1) }\end{array}$ & $\begin{array}{c}2020 \\
\text { (Year 2) }\end{array}$ & $\begin{array}{c}2021 \\
\text { (Year 3) }\end{array}$ & $\begin{array}{c}2022 \\
\text { (Year 4) }\end{array}$ \\
\hline \multirow{3}{*}{ 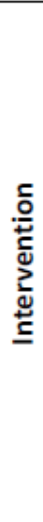 } & $\begin{array}{c}\text { Baseline }(\mathrm{T} 1)= \\
\text { Height, Weight and } \\
\text { Questionnaire } \\
\text { (including HRQoL) }\end{array}$ & $\begin{array}{c}\text { 12-month post (T3) = } \\
\text { Height, Weight and } \\
\text { Questionnaire } \\
\text { (including HRQoL) }\end{array}$ & $\begin{array}{l}\text { 24-month post (T4) = } \\
\text { Height, Weight and } \\
\text { Questionnaire } \\
\text { (including HRQoL) }\end{array}$ & $\begin{array}{c}\text { 36-month post (T5) = } \\
\text { Height, Weight and } \\
\text { Questionnaire } \\
\text { (including HRQoL) }\end{array}$ \\
\hline & $\begin{array}{c}\text { SHINE program } \\
\text { completed over } 8 \text { weeks } \\
\text { during class time }\end{array}$ & $\begin{array}{c}\text { SHINE booster session } \\
\text { completed during one } \\
\text { class }\end{array}$ & $\begin{array}{l}\text { SHINE booster session } \\
\text { completed during one } \\
\text { class }\end{array}$ & $\begin{array}{l}\text { SHINE booster } \\
\text { session completed } \\
\text { during one class }\end{array}$ \\
\hline & $\begin{array}{l}\text { Post-intervention (T2) = } \\
\text { Height, Weight and } \\
\text { Questionnaire } \\
\text { (including HRQoL) }\end{array}$ & & & \\
\hline \multirow{2}{*}{ 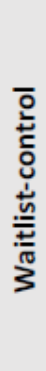 } & $\begin{array}{l}\text { Baseline }(\mathrm{T} 1)= \\
\text { Height, Weight and } \\
\text { Questionnaire } \\
\text { (including HRQoL) }\end{array}$ & $\begin{array}{c}\text { 12-month post (T3) = } \\
\text { Height, Weight and } \\
\text { Questionnaire } \\
\text { (including HRQoL) }\end{array}$ & $\begin{array}{l}\text { 24-month post (T4) = } \\
\text { Height, Weight and } \\
\text { Questionnaire } \\
\text { (including HRQoL) }\end{array}$ & $\begin{array}{c}\text { 36-month post (T5) = } \\
\text { Height, Weight and } \\
\text { Questionnaire } \\
\text { (including HRQoL) }\end{array}$ \\
\hline & $\begin{array}{l}\text { Post-intervention (T2) = } \\
\text { Height, Weight and } \\
\text { Questionnaire } \\
\text { (including HRQoL) }\end{array}$ & & & \\
\hline
\end{tabular}

Figure 1 Timeline for the SHINE-SFA randomised controlled trial. HRQoL,health-related quality of life; SHINE, Supporting Healthy Image, Nutrition and Exercise.

\section{Intervention and comparator}

The SHINE intervention will consist of an online programme, delivered in weekly sessions of approximately $50 \mathrm{~min}$ over eight consecutive weeks during scheduled HPE lessons as per the national curriculum. ${ }^{19}$ The programme consists of four themes on healthy habits-nutrition, physical activity, emotions and body (self) image-and each theme has multiple modules that can be completed in approximately $5 \mathrm{~min}$. In addition to the online programme, data will be collected via questionnaires at baseline (T1), post-intervention (T2), 12 months (T3), 24 months (T4) and 36 months (T5) (figure 1). The questionnaires include screening measures for depressive symptoms and eating disorder symptoms. The measures used are the short form of the Center for Epidemiologic Studies - Depression Scale $\left(\right.$ CESD10 ${ }^{22}$ and the Adolescent version of the Eating Disorder Examination-Questionnaire (EDEA) ${ }^{23}$ Results from these screening measures at baseline will identify participants who are experiencing or are at-risk of having depression, eating disorders or weight issues so that they can receive targeted content in the SHINE intervention. The pathways for completion are self-directed and content is progressively presented, with the targeted content surreptitiously delivered so as to avoid potential stigma or embarrassment. Intervention content is based on an effective programme originally developed for college students in the USA (the 'Staying Fit' programme ${ }^{24} 25$ ), and modified as both a universal and targeted intervention for adolescents in the Australian context. The intervention online content is based on CBT and provides goal setting and suggested self-help strategies to alter unhelpful or unhealthy ways of thinking about common adolescent problems to support behaviour change. Class teachers will receive a training manual on how to access and support the SHINE programme (including a telephone hotline for teachers available during intervention implementation to troubleshoot any potential technical issues). A website comprising of key content from the intervention will be available for parents to access during the course of the intervention, as these have previously been demonstrated to improve parental attitudes towards weight and shape $^{1426}$ and are associated with better outcomes. ${ }^{27}$ Booster SHINE sessions will be delivered to students during one class period in both Grades 8 and 9, 2 and 3 years after commencement of the programme. The study timeline is given in figure 1.

The comparator for the intervention is defined as the waitlist control schools who will receive the usual national HPE curriculum. ${ }^{19}$ Schools will be randomised to intervention or waitlist-control arms using concealed web-based randomisation. Participation will be facilitated by ethics approval for passive consent, enabling all students in Grade 7 at participating schools to receive either the SHINE intervention or standard teacherdelivered curriculum. The only students who will not be captured are those with written opt-out/non-consent from parents. Student assent will also be collected at each assessment. 


\section{Sample size and missing data}

We will recruit a minimum of 30 schools from metropolitan and regional Victoria. Assuming a high enrolment rate associated with the opt-out consent approach ${ }^{28}$ we expect to enrol an average of 150 seventh-grade children per school, that is, a total of 4500 participants (approximately 2250 in each group). Considering 20\% attrition, we aim to collect complete data on 3600 participants (1800 per group). This sample size provides $80 \%$ power of detecting the following effect sizes at 36 months $(\alpha=0.05$ and two-sided tests) after allowing for clustering of students in classes with the specified design effect (DE), assuming the average number of participating students per class will be 20 .

\section{Overweight and obesity prevalence}

Assuming a $30 \%$ prevalence of overweight and obesity in the control group at 36 month ${ }^{29}$ a sample of 3600 students allows to detect a $5.8 \%$ point reduction in the prevalence in the intervention group at 36 months (assuming Intraclass Correlation Coefficient $($ ICC $)=0.05, \mathrm{DE}=1.95$ ) or a $4.9 \%$ point reduction $(\mathrm{ICC}=0.02, \mathrm{DE}=1.38) .{ }^{30}$

\section{BMl z-score}

Assuming a conservative $30 \%$ prevalence of overweight or obesity at baseline, we would have a sample of 540 such students in each arm. Assuming a SD of 0.38 for BMI z-scores at 36 months in this group (informed by our previous study), we would be able to detect a difference of 0.05 points on the BMI z-score (assuming ICC $=0.05$, $\mathrm{DE}=1.95)$ or 0.04 points $(\mathrm{ICC}=0.02, \mathrm{DE}=1.38)$.

\section{Outcome measures}

Study outcome measures relevant to the economic evaluation are presented in table 1. Online questionnaires will be used to collect all outcome data except height and weight, which will be measured by trained research assistants at baseline, and at 8 weeks and 12, 24 and 36 months from baseline.

The primary outcome measure for the within-trial CEA will be the change in BMI at 36 months in the intervention group, as compared with the waitlist-control group. The secondary outcome measure for within-trial CEA will

\begin{tabular}{ll}
\hline $\begin{array}{l}\text { Table } 1 \text { Summary of outcome measures for economic } \\
\text { evaluation }\end{array}$ \\
\hline Study outcome measure & Method for data collection \\
\hline BMI, BMI z-score & $\begin{array}{l}\text { Objectively measured height } \\
\text { and weight by trained research } \\
\text { assistants. BMI z-score estimated } \\
\text { using the WHO reference standard. }\end{array}$ \\
Quality of life & $\begin{array}{l}\text { Online questionnaire using the } \\
\text { AQoL-6D }\end{array}$ \\
Physical activity & $\begin{array}{l}\text { Online questionnaire using the } \\
\text { APARQ }\end{array}$ \\
\hline
\end{tabular}

$A P A R Q$, adolescent physical activity recall questionnaire; AQoL$6 \mathrm{D}$, assessment of quality of life 6D; BMI, body mass index. include the time spent in physical activity in the intervention group, as compared with the waitlist-control group at 12, 24 and 36 months. CUA will also be undertaken using the difference in quality of life in the intervention group, as referenced to the waitlist-control group at 12, 24 and 36 months. Quality of life will be measured using the adolescent version of the Assessment of Quality of Life 6D (AQoL-6D), a preference-based multi-attribute utility instrument comprised of 20 items from six dimensions (independent living, relationships, mental health, coping, pain and senses) with an adolescent-specific scoring algorithm. ${ }^{31}$ The items from the six dimensions comprising the adolescent version of the AQoL-6D will be used as the outcome measure for evaluation and utility values will be adjusted for the adolescent population using the adolescent specific preference weights. ${ }^{31}$

\section{Resource use and intervention costs}

Given the intervention is delivered in the school setting, the economic evaluation will be conducted from the public payer perspective. The public payer perspective was chosen as both education and healthcare are publicly, universally provided in Australia. The evaluation will be undertaken assuming 'steady state' conditions (ie, the intervention is assumed to be running at full effectiveness and costs associated with the programme's development will not be included into the analyses). Incremental costs from resource use associated with implementation of the intervention will be identified using pathway analysis. The research team will collect resource use data alongside the trial (up to 36 months post intervention) using a standardised tool created in Microsoft Excel and a survey of teacher time. Study invoices, receipts and published values will be used for costing, with all costs measured in 2021 Australian dollars. Costs will be reported as total cost of the intervention, as well as mean cost per participant. Where appropriate, costs and consequences will be discounted at the commonly accepted $3 \%$ discount rate. ${ }^{32}$ Table 2 provides a summary of the resource use and intervention costs to be collected alongside the SHINE cRCT.

\section{Within-trial cost-effectiveness and cost-utility analyses}

The within-trial CEA and CUA reference year will be 2021 and will include total costs and effects that accumulate during the intervention period (up to 36 months from baseline). Results will be presented as the cost per BMI unit saved, cost per extra minute of physical activity gained and the cost per QALY gained. Incremental costeffectiveness ratios (ICERs) will be calculated as the difference in costs between the intervention and the comparator divided by the difference in benefit.

\section{Modelled cost-utility analysis}

The modelled CUA will assume that the intervention is integrated in national curriculum and will include costs accumulated during the intervention implementation phase and effects that accumulate over a longer time horizon, assumed to be rest-of-life or 100 years of age. 
Table 2 Resource use and intervention costs collected alongside the SHINE cRCT

\begin{tabular}{lll}
\hline Cost category & Costs & Collection strategy and source of data \\
\hline Time costs & $\begin{array}{l}\text { Costs associated with training teachers on intervention } \\
\text { delivery. Costs associated with lesson planning. Salary } \\
\text { on-costs will be included. }\end{array}$ & $\begin{array}{l}\text { Teacher time use survey } \\
\text { Published salary values from Government } \\
\text { websites }^{47} 48\end{array}$ \\
Travel costs & $\begin{array}{l}\text { Costs associated with travel for training or intervention- } \\
\text { related purposes. }\end{array}$ & $\begin{array}{l}\text { Project administrative records } \\
\text { Published values from Government websites }\end{array}$ \\
Equipment costs & $\begin{array}{l}\text { Costs include teacher training materials, printing, and so } \\
\text { on. Consumables/materials costs. }\end{array}$ & Project administrative records \\
\hline
\end{tabular}

Costs are estimated assuming steady-state intervention (ie, excluding intervention development costs). $\mathrm{cRCT}$, cluster randomised control trial; SHINE, Supporting Healthy Image, Nutrition and Exercise.

Costs and effects will be extrapolated to reflect intervention delivery to the Australian population of Grade 7 students in all Government and non-Government schools.

A proportional multi-state, multiple cohort life table Markov model developed as part of an obesity priority setting study in Australia (the ACE-Obesity Policy model) will be updated and used to estimate the obesity and physical activity-related health outcomes and healthcare cost-savings of the SHINE intervention. ${ }^{33}$ The reference year will be 2018 for the modelled CUA, due to the availability of epidemiological data to inform the model. The model uses the 'relative risk shift' method for the calculation of population impact fractions ${ }^{34}$ to estimate the consequences of a change in BMI or a change in physical activity on the incidence of related diseases. The obesityrelated diseases included in the model are ischaemic heart disease, hypertensive heart disease, ischaemic stroke, diabetes, colorectal cancer, kidney cancer, breast cancer, endometrial cancer and osteoarthritis. Physical activity-related diseases include ischaemic heart disease, stroke, type 2 diabetes, breast cancer and colon cancer, with an adjustment factor to avoid double-counting for diseases where both obesity and physical inactivity are risk factors. ${ }^{35}$ Cohort-based modelling will allow for the disease-related benefits not present in adolescence to be estimated assuming lingering BMI effects. Differences in the health-related quality of life between healthy weight and obese adolescents will be incorporated using utility values derived from the study population using the AQoL-6D Adolescent version.

The change in risk arising from a change in BMI and/ or physical activity will be compared against the counterfactual scenario, where the distributions of BMI and/or physical activity in the 2018 Australian population (ie, the reference population) remain unchanged. The model will use data from the Australian Health Survey 2017$2018^{2}$ and disease epidemiology from the Global Burden of Disease study ${ }^{36}$ (table 3). Healthcare costs will be estimated using data from the Australian Institute of Health and Welfare, adjusted to 2018 values using the health price index ${ }^{37}$ Modelling will be undertaken in Microsoft Excel and results will be presented as cost per life year saved, cost per health-adjusted life years gained (HALYs gained), healthcare cost-savings from diseases averted and mean ICER assuming maintenance of intervention effect over the cohort's lifetime. Results will be presented on a cost-effectiveness plane, where interventions that are both health-promoting and cost-saving are considered 'dominant'.

\section{Uncertainty and sensitivity analyses}

The Microsoft Excel add-in Ersatz ${ }^{38}$ will be used to estimate the $95 \%$ uncertainty intervals around epidemiological probabilities and cost estimates using Monte Carlo simulation (2000 runs). Sensitivity analyses will be undertaken for the modelled CUA by varying key assumptions around the sustainability of the intervention effect. Sensitivity analyses will also vary the discount rate to $0 \%$ and $5 \%$ as per recommended guidelines. ${ }^{39}$

\section{Qualitative analysis}

Semi-structured interviews will be conducted with key intervention stakeholders to explore the potential for scalability of the intervention, and the implications for costs, effects and cost-effectiveness of the intervention if delivered at scale. Key intervention stakeholders $(n=20$; defined as potential programme providers (eg, education department and curriculum authority representatives), programme deliverers (eg, teachers and principals) and lead intervention researchers (eg, Chief and Associate Investigators,

Table 3 Input parameters for health impact modelling

\begin{tabular}{ll} 
Parameters & $\begin{array}{l}\text { Data source and } \\
\text { assumptions }\end{array}$ \\
\hline $\begin{array}{l}\text { Total population estimates } \\
\text { (population numbers, mortality } \\
\text { rates, BMI distribution, PA levels) }\end{array}$ & $\begin{array}{l}\text { Australian Bureau of } \\
\text { Statistics }\end{array}$ \\
$\begin{array}{l}\text { Disease epidemiology, disability } \\
\text { weights }\end{array}$ & $\begin{array}{l}\text { Institute for Health } \\
\text { Metrics and Evaluation }\end{array}$ \\
$\begin{array}{l}\text { Relative risks of PA-related } \\
\text { diseases by risk categories }\end{array}$ & $\begin{array}{l}\text { Zapata-Diomedi et al } \\
\text { 2016 }\end{array}$ \\
$\begin{array}{l}\text { Relative risks, total years of life } \\
\text { lived with disability }\end{array}$ & $\begin{array}{l}\text { Institute for Health } \\
\text { Metrics and Evaluation }\end{array}$ \\
Disease healthcare costs & $\begin{array}{l}\text { Australian Institute of } \\
\text { Health and Welfare }\end{array}$ \\
\hline
\end{tabular}

BMI, body mass index; PA, physical activity. 
Project Manager)) will be purposively sampled and invited to participate in face-to-face or telephone interviews. The interviews will be guided by the Knowledge-To-Action framework ${ }^{40}$ and the Consolidated Framework for Implementation Research ${ }^{41}$ and will be recorded and transcribed verbatim. Data will be analysed thematically using NVivo 12 software to assist with data coding, and reported as per guidelines. ${ }^{42}$ Data from the interviews will be summarised narratively, ${ }^{43}$ and will also inform further sensitivity analysis of the modelled CUA by adjusting the cost and effect estimates used to determine cost-effectiveness at scale according to qualitative findings.

\section{Approvals and registration}

The SHINE RCT has been registered with the Australian New Zealand Clinical Trials Registry.

\section{Ethics and dissemination}

Ethics approval for the SHINE study has been received from the Deakin University Human Research Ethics Committee (\#2017-269) and the Victorian Department of Education and Training (\#2018_003630). Data pertaining to the economic evaluation of the intervention will be held on secure servers at Deakin University, Australia, as per ethics requirements. Publications planned using this data include a peer-reviewed journal article on the cost-effectiveness of the intervention at 36 months and a peer-reviewed journal article exploring the potential impact on costs, effects and cost-effectiveness of scale-up. Findings will be disseminated both within academia, to the participating schools and to policymakers. Study findings will be placed on the project website (https://www.deakin.edu.au/cphr/our-research/ epidemiology-unit/research-projects/shine) to enable viewing by study participants and their parents.

\section{STRENGTHS}

Results from the within trial CEA and CUA will be supplemented with results from a modelled CUA, examining the broader potential for cost-effectiveness of the intervention by extrapolating costs and effects to a wider population and modelling over a longer time horizon. In addition, mixed methods research will explore the potential impact on costs, effects and cost-effectiveness should the SHINE intervention be scaled up in future. The results from the proposed economic evaluation will add to the relatively limited evidence base on the 'value for money' of obesity prevention interventions in adolescents, and will provide useful information to decision makers both within the health and education sectors on the economic case for more widespread implementation of the intervention.

\section{LIMITATIONS}

A potential limitation of the study may be missing data on teacher time costs and outcome measures. Missing data will be handled according to published guidelines ${ }^{4}$ and multiple imputation approaches will be used. For
AQoL-6D missing data we will impute values within each dimension as per developer guidelines. Sensitivity analyses will be used to assess the impact of the handling of missing data on study findings. The modelled cost-utility analysis will also be subject to several limitations, including the assumptions used for extrapolating the outcomes and costs from a short-term trial to longer-term healthcare cost-savings and health benefits and the published limitations of the ACE-Obesity Policy model. ${ }^{33}$ Sensitivity analyses will be used to assess the impact of assumptions on overall study findings.

\section{Author affiliations}

${ }^{1}$ Deakin University, Geelong, Deakin Health Economics, Institute for Health Transformation, Geelong, Victoria, Australia

${ }^{2}$ Deakin University, Geelong, Institute for Health Transformation, School of Health and Social Development, Geelong, Victoria, Australia

${ }^{3}$ Department of Paediatrics, The University of Melbourne; Centre for Adolescent Health Royal Children's Hospital, Melbourne, Victoria, Australia

${ }^{4}$ Deakin University, Geelong, Faculty of Health, Geelong, Victoria, Australia

${ }^{5}$ Deakin University, Geelong, School of Information Technology, Geelong, Victoria, Australia

${ }^{6}$ Deakin University, Geelong, Institute for Physical Activity and Nutrition, Faculty of Health, Geelong, Victoria, Australia

${ }^{7}$ School of Medicine, Washington University in St. Louis, Missouri, Missouri, USA

\section{Twitter Victoria Brown @Vicki_BBB}

Contributors JW, SS, MM, WL, KDH, LO and DEW conceived the SHINE study. VB and $\mathrm{MM}$ conceptualised the economic evaluation. VB, MM and LM developed the data collection methodology for the economic evaluation. VB wrote the draft study protocol. All authors reviewed the paper.

Funding This work was supported by a National Health and Medical Research Council (NHMRC) project grant (1122840) and a Deakin University School of Health and Social Development School Grant (2019-SRG006). VB is supported by a Deakin University Postdoctoral Research Fellowship.

Competing interests None declared.

Patient and public involvement Patients and/or the public were not involved in the design, or conduct, or reporting, or dissemination plans of this research.

Patient consent for publication Not required.

Provenance and peer review Not commissioned; externally peer reviewed.

Open access This is an open access article distributed in accordance with the Creative Commons Attribution Non Commercial (CC BY-NC 4.0) license, which permits others to distribute, remix, adapt, build upon this work non-commercially, and license their derivative works on different terms, provided the original work is properly cited, appropriate credit is given, any changes made indicated, and the use is non-commercial. See: http://creativecommons.org/licenses/by-nc/4.0/.

ORCID iD

Victoria Brown http://orcid.org/0000-0003-2891-9476

\section{REFERENCES}

1 Abarca-Gómez L, Abdeen ZA, Hamid ZA, et al. Worldwide trends in body-mass index, underweight, overweight, and obesity from 1975 to 2016: a pooled analysis of 2416 population-based measurement studies in 128.9 million children, adolescents, and adults. The Lancet 2017;390:2627-42

2 Australian Bureau of Statistics. National health survey 2017-18 Canberra, Australia: ABS, 2018. Available: https://www.abs.gov.au/ AUSSTATS/abs@.nsf/allprimarymainfeatures/F6CE5715FE4AC1B1 CA257AA30014C725?opendocument

3 Australian Institute of Health and Welfare. Risk factors to health Canberra. Australia: AlHW, 2017. https://www.aihw.gov.au/reports/ biomedical-risk-factors/risk-factors-to-health/contents/insufficientphysical-activity 
4 Johnson B, Bell L, Zarnowiecki D, et al. Contribution of discretionary foods and drinks to Australian children's intake of energy, saturated fat, added sugars and salt. Children 2017;4:104.

5 Reilly JJ, Methven E, McDowell ZC, et al. Health consequences of obesity. Arch Dis Child 2003;88:748-52.

6 Narang I, Mathew JL. Childhood obesity and obstructive sleep apnea. J Nutr Metab 2012;2012:1-8.

7 Egan KB, Ettinger AS, Bracken MB. Childhood body mass index and subsequent physician-diagnosed asthma: a systematic review and meta-analysis of prospective cohort studies. BMC Pediatr 2013;13:121.

8 Simmonds M, Llewellyn A, Owen CG, et al. Predicting adult obesity from childhood obesity: a systematic review and meta-analysis. Obes Rev 2016;17:95-107.

9 World Health Organisation. Report of the commission on ending childhood obesity. Geneva: WHO, 2016.

10 Patton GC, Johnson-Sabine E, Wood K, et al. Abnormal eating attitudes in London schoolgirls--a prospective epidemiological study: outcome at twelve month follow-up. Psychol Med 1990;20:383-94.

11 Moores CJ, Bell LK, Miller J, et al. A systematic review of community-based interventions for the treatment of adolescents with overweight and obesity. Obes Rev 2018;19:698-715.

12 Waters E, de Silva-Sanigorski A, Burford BJ, et al. Interventions for preventing obesity in children. Cochrane Database Syst Rev 2011;165.

13 Brown T, Moore THM, Hooper L, et al. Interventions for preventing obesity in children. Cochrane Database Syst Rev 2019;14.

14 Jones M, Luce KH, Osborne MI, et al. Randomized, controlled trial of an internet-facilitated intervention for reducing binge eating and overweight in adolescents. Pediatrics 2008;121:453-62.

15 Beintner I, Jacobi C, Taylor CB. Effects of an internet-based prevention programme for eating disorders in the USA and Germany-a meta-analytic review. Eur Eat Disord Rev 2012;20:1-8.

16 Lobstein T, Jackson-Leach R, Moodie ML, et al. Child and adolescent obesity: part of a bigger picture. Lancet 2015;385:2510-20.

17 McCrabb S, Lane C, Hall A, et al. Scaling-up evidence-based obesity interventions: a systematic review assessing intervention adaptations and effectiveness and quantifying the scale-up penalty. Obes Rev 2019;20:964-82.

18 Dopp AR, Mundey P, Beasley LO, et al. Mixed-method approaches to strengthen economic evaluations in implementation research. Implement Sci 2019;14:2.

19 Australian Curriculum Assessment and Reporting Authority. Australian curriculum Sydney. Australia: ACARA, 2018. https://www. australiancurriculum.edu.au/f-10-curriculum/health-and-physicaleducation/

20 George B, Harris A, Mitchell A. Cost-effectiveness analysis and the consistency of decision making. Pharmacoeconomics 2001;19:1103-9.

21 Husereau D, Drummond M, Petrou S, et al. Consolidated Health Economic Evaluation Reporting Standards (CHEERS)--explanation and elaboration: a report of the ISPOR health economic evaluation publication guidelines good reporting practices task force. Value Health 2013;16:231-50.

22 Bradley KL, Bagnell AL, Brannen CL. Factorial validity of the center for epidemiological studies depression 10 in adolescents. Issues Ment Health Nurs 2010;31:408-12.

23 White HJ, Haycraft E, Goodwin H, et al. Eating disorder examination questionnaire: factor structure for adolescent girls and boys. Int $J$ Eat Disord 2014;47:99-104.

24 Jones M, Taylor Lynch K, Kass AE, et al. Healthy weight regulation and eating disorder prevention in high school students: a universal and targeted web-based intervention. J Med Internet Res 2014;16:e57.

25 Stanford Online. Staying fit USA: Stanford school of medicine, 2019 Available: https://online.stanford.edu/courses/som-y0014-staying-fit

26 Bruning Brown J, Winzelberg AJ, Abascal LB, et al. An evaluation of an internet-delivered eating disorder prevention program for adolescents and their parents. J Adolesc Health 2004;35:290-6.

27 White MA, Martin PD, Newton RL, et al. Mediators of weight loss in a family-based intervention presented over the Internet. Obes Res 2004;12:1050-9.

28 Strugnell C, Orellana L, Hayward J, et al. Active (Opt-In) consent underestimates mean BMI-z and the prevalence of overweight and obesity compared to passive (Opt-Out) consent. Evidence from the healthy together Victoria and childhood obesity study. Int J Environ Res Public Health 2018;15:ijerph15040747.

29 Williams JW, Canterford L, Toumbourou JW, et al. Social development measures associated with problem behaviours and weight status in Australian adolescents. Prev Sci 2015;16:822-31.

30 Robb W, lachan R, eds. Design effects in school surveys in the online proceedings of the survey research methods section: american statistical association, 2002.

31 Moodie M, Richardson J, Rankin B, et al. Predicting time trade-off health state valuations of adolescents in four Pacific countries using the assessment of quality-of-life (AQoL-6D) instrument. Value Health 2010;13:1014-27.

32 Gold M, Siegel J, Russell L, et al. Cost effectiveness in health and medicine. Oxford: Oxford University Press, 1996.

33 Ananthapavan J, Sacks G, Brown V, et al. Assessing costeffectiveness of obesity prevention policies in Australia 2018 (ACEObesity policy). Melbourne: Deakin university, 2018.

34 Barendregt JJ, Veerman JL. Categorical versus continuous risk factors and the calculation of potential impact fractions. J Epidemiol Community Health 2010;64:209-12.

35 Cobiac L, Vos T, Veerman L. Cost-Effectiveness of weight Watchers and the Lighten up to a healthy lifestyle program. Aust N Z J Public Health 2010;34:240-7.

36 Institute for Health Metrics and Evaluation. Global burden of disease database Seattle: IHME, University of Washington, 2016. Available: http://www.healthdata.org/search-gbd-data?s=Transport\%20injuries

37 Australian Institute of Health and Welfare. Disease expenditure in Australia. Canberra, Australia: AlHW, 2019.

38 EpiGear International. Ersatz Brisbane, Australia: EpiGear international, 2016. Available: http://www.epigear.com/index_files/ ersatz.html

39 Australian Government Department of Health. Guidelines for preparing submissions to the pharmaceutical benefits advisory committee (PBAC), version 5.0. Canberra: Department of Health, 2016.

40 Graham ID, Logan J, Harrison MB, et al. Lost in knowledge translation: time for a MAP? J Contin Educ Health Prof 2006;26:13-24

41 Damschroder LJ, Aron DC, Keith RE, et al. Fostering implementation of health services research findings into practice: a consolidated framework for advancing implementation science. Implement Sci 2009;4:50.

42 Tong A, Sainsbury P, Craig J. Consolidated criteria for reporting qualitative research (COREQ): a 32-item checklist for interviews and focus groups. Int J Qual Health Care 2007;19:349-57.

43 Braun V, Clarke V. Using thematic analysis in psychology. Qual Res Psychol 2006;3:77-101.

44 Faria R, Gomes M, Epstein D, et al. A guide to handling missing data in cost-effectiveness analysis conducted within randomised controlled trials. Pharmacoeconomics 2014;32:1157-70.

45 Richardson JRJ, Peacock SJ, Hawthorne G, et al. Construction of the descriptive system for the assessment of quality of life AQoL-6D utility instrument. Health Qual Life Outcomes 2012;10:38.

46 Booth ML, Okely AD, Chey TN, et al. The reliability and validity of the adolescent physical activity recall questionnaire. Med Sci Sports Exerc 2002;34:1986-95.

47 Deakin University. Salary benefits Melbourne, Australia: Deakin university, 2018. Available: http://www.deakin.edu.au/about-deakin/ work-at-deakin/why-work-at-deakin/staff-benefits/salary-benefits

48 Victorian Department of Education and Training. Salary rates Melbourne, Victoria: DET, 2019. Available: https://www.education.vic. gov.au/hrweb/employcond/Pages/salaries.aspx

49 Australian Taxation Office. Motor vehicle expenses Canberra, Australia: ATO, 2018. Available: https://www.ato.gov.au/Business/ Income-and-deductions-for-business/Deductions/Motor-vehicleexpenses/

50 Australian Bureau of Statistics.. 2016 census Canberra, Australia: ABS, 2017. Available: http://www.abs.gov.au/websitedbs/ censushome.nsf/home/2016

51 Institute for Health Metrics and Evaluation. Global burden of disease study 2017 (GBD 2017). Disability Weights Washington: IHME, 2019. http://ghdx.healthdata.org/record/ihme-data/gbd-2017-disabilityweights

52 Zapata-Diomedi B, Herrera AMM, Veerman JL. The effects of built environment attributes on physical activity-related health and health care costs outcomes in Australia. Health Place 2016;42:19-29. 